\title{
Emotion Comprehension in Intramodal and Cross-modal Matching: A Preliminary Comparison between Children With Autism Spectrum Disorders and Those With Williams Syndrome
}

\author{
Soichiro Matsuda ${ }^{1,2, *}$ and Junichi Yамамото ${ }^{1,3}$ \\ ${ }^{1}$ Department of Psychology, Keio University, Tokyo, Japan \\ ${ }^{2}$ Japan Society for the Promotion of Science, Tokyo, Japan \\ ${ }^{3}$ CREST, Japan Science and Technology Agency, Saitama, Japan
}

\begin{abstract}
Autism spectrum disorders (ASD) and Williams syndrome (WS) exhibit contrasting forms of emotion comprehension; however, few studies have directly compared the two. In this study, we directly compared six children with ASD, chronological age 3-7, with six children with WS, chronological ages ranging 4-11, but were matched with the ASD children in terms of developmental age. The children's development of emotion comprehension was assessed by intramodal (visual stimulus-visual stimulus) and cross-modal (auditory stimulus-visual stimulus) matching. Facial expressions were used as visual stimuli, and affective prosodies were used as auditory stimuli. Children with ASD were less accurate than those with WS on cross-modal matching, but equally accurate on intramodal matching. The results suggest that deficits in emotion comprehension among children with ASD may be related to difficulties in understanding the relationship between auditory and visual stimuli and the deficits may be greater at younger ages.
\end{abstract}

Key Words: emotion comprehension, facial expression, affective prosody, autism spectrum disorders, Williams syndrome

\section{Introduction}

Autism spectrum disorders (ASD) and Williams syndrome (WS) are neurodevelopmental disorders characterized by distinctive social behaviors. Individuals with ASD have social deficits, particularly in their comprehension of others' emotions as conveyed by nonverbal cues, such as facial expressions or affective prosodies (Gross, 2008; Kuusikko, Haapsamo, Jansson-Verkasao, Hurtig, Mattila, Ebeling, Jussila, Bölte, \& Moilanen, 2009; Wallace, Coleman, \& Bailey, 2008). On the other hand, individuals with WS are highly social (Klein-Tasman \& Mervis, 2003). They recognized others' emotions to the same extent as

\footnotetext{
* Corresponding Author

Mailing Address: 2-15-45 Mita, Minato-ku, Tokyo 108-8345, Japan

E-mail Address: atom.opens.the.stargate@gmail.com

Received December 3, 2014, Accepted August 29, 2015

DOI: 10.6033/specialeducation.4.1
}

or even more than IQ-matched controls (Gagliardi, Frigerio, Burt, Cazzaniga, Perrett, \& Borgatti, 2003; Plesa-Skwerer, Faja, Schofield, Verbalis, \& Tager-Flusberg, 2006a; Plesa-Skwerer, Verbalis, Schofield, Faja, \& Tager-Flusberg, 2006b). Although these two disorders exhibit contrasting forms of emotion comprehension, few studies have investigated the differences between them, especially in children. Therefore, the early development of emotion comprehension in these groups has remained largely unknown.

As pointed out by Tager-Flusberg, Plesa-Skwerer, and Joseph (2006), investigating differences between ASD and WS contribute to a better understanding of social behavior with specific focus on emotion comprehension. Even though ASD and WS are characterized by their social behavior, there is variability within each neurodevelopmental disorder. Some studies have found atypical emotion comprehension in individuals with ASD (Bormann-Kischkel, Vilsmeier, \& Baude, 1995; Kuusikko et al., 2009) or WS (Gagliardi et al., 2003; Setter, Stojanovik, van Ewijk, \& 
Moreland, 2007). Other studies have not found such atypical emotion comprehension (Capps, Yirmiya, \& Sigman, 1992; Castelli, 2005; Plesa-Skwerer et al., 2006a; Plesa-Skwerer et al., 2006b). This variability may have occurred due to the use of different stimuli and responses (Harms, Martin, \& Wallace, 2010; Tager-Flusberg et al., 2006).

Based on the modality of stimuli and responses used, previous studies that have investigated emotion comprehension can be divided into two types: intramodal (i.e., visual stimulus-visual stimulus) and cross-modal (i.e., auditory stimulus-visual stimulus) matching (Matsuda \& Yamamoto, 2013, 2014, 2015). In intramodal matching, facial expressions were presented as sample stimuli and either facial expressions or texts were used as response choices. In crossmodal matching, affective prosodies representing different emotional states were presented as sample stimuli and facial expressions or texts were used as response choices.

To date, only one study (Lacroix, Guidetti, Rogé, \& Reilly, 2009) has used intramodal matching to compare children with ASD and those with WS in emotion comprehension. In that study, pictures of facial expressions were used as both sample stimuli and response choices. Lacroix et al. (2009) found no difference in the rate of correct responses in intramodal matching between children with ASD and those with WS. However, cross-modal matching in children with ASD and those with WS has not yet been examined.

Recent studies have indicated that cross-modal matching is more difficult than intramodal matching for children with ASD. A study using pictures of facial expressions as both samples and response choices found no significant deficits in children with ASD (Castelli, 2005), and another study revealed deficits in children with ASD when affective prosodies were used as sample stimuli, and illustrations were used as response choices (Peppé, McCann, Gibbon, O’Hare, \& Rutherford, 2007).

It is possible that the difficulties experienced by children with ASD in cross-modal matching of emotion comprehension are related to their age. In older children (aged 12-16) with ASD, studies have not found deficits (Baker, Montgomery, \& Abramson, 2010; Grossman, Bemis, Plesa-Skwerer, \& Tager-Flusberg, 2010; Jones, Pickles, Falcaro, Marsden, Happé, Scott, Sauter, Tregay, Phillips, Baird, Simonoff, \& Charman, 2011; Paul, Augustyn, Klin, \& Volkmer,
2005). On the other hand, children aged 4-10 with ASD have shown significant impairments (Linder \& Rosén, 2006; Matsuda \& Yamamoto, 2015; Peppé et al., 2007). These results indicate that cross-modal matching is difficult especially for younger children with ASD.

For children with WS, one study has used intramodal matching, but no studies have been conducted using cross-modal matching (Plesa-Skwerer et al., 2006a). A study that used pictures of facial expressions as both samples and response choices found no deficits in children with WS (Tager-Flusberg \& Sullivan, 2000). This result suggests that intramodal matching is not difficult for children with WS.

The present study compared the intramodal and cross-modal matching of emotion comprehension in children with ASD and those with WS. To this end, we used facial expressions as visual stimuli and affective prosodies as auditory stimuli. The study focused on the participants who are younger in both chronological and developmental age than the participants in previous studies conducted with these groups.

\section{Method}

\section{Participants}

Six children with ASD participated in the study. Five children were diagnosed with autistic disorder, and one child was diagnosed with pervasive development disorder not otherwise specified (PDD-NOS). Diagnoses were provided by an outside professional according to the criteria of the DSM-IV-TR (American Psychiatric Association, 2000). The standardized diagnostic scales used in the study, such as the Autism Diagnostic Interview-Revised (ADI-R; Le Couteur, Rutter, Lord, Rios, Robertson, Holdgrafer, \& McLennan, 1989; Lord, Rutter, \& Le Couteur, 1994) and the Autism Diagnostic Observation Schedule (ADOS; Lord, Rutter, DiLavore, \& Risi, 2002) have not been officially translated into Japanese or adapted to the Japanese culture. Therefore, confirming outside clinical diagnoses of ASD using the Childhood Autism Rating Scale (CARS; Schopler, Reichler, DeVellis, \& Daly, 1980) was necessary. The CARS classified three children as mild to moderately autistic and the other three as severely autistic. The chronological ages ranged from 3 years 7 months to 7 years 4 months (mean 5 years 9 months), and the developmental ages ranged from 2 years 2 months to 6 
Intramodal and Cross-modal Matching in Emotion

Table 1 Participant Characteristics

\begin{tabular}{|c|c|c|c|c|c|}
\hline Child & Chronological age (year) & Developmental age $^{\mathrm{a}}$ (year) & Gender & Autism severity ${ }^{\mathrm{b}}$ & Diagnosis \\
\hline 1 & 7.3 & 2.2 & Boy & 49.5 & Autism \\
\hline 2 & 4.0 & 2.9 & Boy & 40 & PDD-NOS \\
\hline 3 & 3.6 & 2.9 & Boy & 36 & Autism \\
\hline 4 & 5.6 & 3.4 & Boy & 42 & Autism \\
\hline 5 & 7.0 & 5.3 & Boy & 30.5 & Autism \\
\hline 6 & 7.3 & 6.6 & Boy & 34.5 & Autism \\
\hline 7 & 4.2 & 2.2 & Girl & $\mathrm{N} / \mathrm{A}$ & Williams syndrome \\
\hline 8 & 5.2 & 2.4 & Boy & $\mathrm{N} / \mathrm{A}$ & Williams syndrome \\
\hline 9 & 5.0 & 2.8 & Girl & $\mathrm{N} / \mathrm{A}$ & Williams syndrome \\
\hline 10 & 7.0 & 2.8 & Girl & N/A & Williams syndrome \\
\hline 11 & 10.0 & 4.9 & Boy & N/A & Williams syndrome \\
\hline 12 & 11.4 & 5.9 & Girl & N/A & Williams syndrome \\
\hline
\end{tabular}

years 6 months (mean 3 years 10 months). The children's developmental age (DA) was assessed using the Kyoto Scale of Psychological Development 2001 (KSPD; Ikuzawa, Matsushita, \& Nakase, 2002). This assessment calculates the average scores for the subitems of physical-motion, verbal-social, cognitiveadaptation, and total developmental age. The KSPD was created for use with typically developing infants and low-functioning children with ASD or other developmental disorders. The WS group was matched with the ASD group on the basis of developmental age. All the children in the WS group were diagnosed with WS from a clinician, and this diagnosis had been confirmed by means of the Fluorescent In Situ Hybridization test. Chronological ages ranged from 4 years 2 months to 11 years 5 months (mean 7 years 1 month), and the developmental ages ranged from 2 years 2 months to 5 years 10 months (mean 3 years 5 months). Table 1 shows the characteristics of the children.

\section{Stimuli}

The stimulus set used was based on the study by Matsuda and Yamamoto (2015). The stimuli have been validated in typical adults (Matsuda and Yamamoto, 2015).

Visual stimuli (facial expressions). Eight color pictures of male faces were used, of which four pictures of each of the two men showed four emotions (happy, surprised, angry, and sad). Visual stimuli were printed and laminated on cards $(9.5 \mathrm{~cm}$ by $13.0 \mathrm{~cm}$ ).

Auditory stimuli (affective prosodies). A male experimenter stated a word, "sensei" ("teacher" in Japanese) in a happy, surprised, angry, or sad voice. Sample recordings were rated prior to the experiment.

\section{Procedure}

We evaluated two experimental tasks: intramodal (facial expression-facial expression) and cross-modal (affective prosody-facial expression) matching. The intramodal matching required the children to match the pictures of facial expressions across different facial identities. The cross-modal matching required them to match an affective prosody to the corresponding facial expression.

We tested each child individually in a testing room at Keio University. We provided the children and their caregivers with an IRB-approved informed consent form that explained the study, and the caregivers signed the consent form. The children sat in a chair facing the desk, and the experimenter sat opposite the child.

The children were presented with eight training trials and 20 test trials for each part of the experiment (i.e., intramodal and cross-modal matching). After completion of the eight training trials, the 20 test trials followed immediately. The four sample stimuli were presented twice each for the training trials, and the stimuli were then presented five times each for 
the test trials, in a semi-random order. For each trial, the four response choices were placed on the desk in a horizontal alignment. The positions of the response choices were changed twice during the test trials.

Intramodal matching (facial expression-facial expression). (i) Training trials. The training trials were conducted to assess the children's ability to identify the facial expressions. During the training trial, only one model's facial expression was used; the sample stimulus and response choices displayed the same model's facial expression. The child was presented a picture of a facial expression and was instructed to find the same expression among the response choices and then to point at it or hand it to the experimenter. For each response given by the child, no differential feedback was provided by the experimenter. The child was given approximately five seconds to make an initial response. If there was no response, the experimenter presented the sample stimulus again. After the child responded to the second presentation or if the child still did not respond after approximately five seconds the experimenter continued on immediately to the next trial. Correct responses were recorded when the child chose the corresponding response choice; incorrect responses were recorded when the child selected a different response choice or did not respond at all within approximately five seconds after the second presentation of the sample stimulus. If the child failed to provide any correct responses during the eight training trials, the procedure was terminated. (ii) Test trials. During the test trial, two models' facial expressions were used. One was used as a sample stimulus and the other as a response choice. All other procedures were the same as that in the training trials.

Cross-modal matching (affective prosody-facial expression). The procedure was essentially the same as in intramodal matching, except for the stimuli used. (i) Training trials. Training trials were conducted to assess the children's ability to understand the cross-modal matching task. Sample stimuli were four animal calls (dog, cat, pig, and chicken), and the response choices were picture cards corresponding to each of the animals. The child was presented with an animal call in the experimenter's voice (e.g., "wanwan," which is "bow-wow" in Japanese) and was instructed to find the corresponding response choice and then to point at it or hand it to the experimenter. (ii) Test trials. During the test trial, a word spoken with the affective prosody was used as the sample stimulus, and the pictures of facial expressions were used as response choices. The experimenter hid his face behind a blackboard when presenting a sample stimulus.

\section{Statistical Analysis}

The analyses were performed by using the Statistical Package for Social Sciences (SPSS/Win in 22.0). Due to some violations of parametric testing (small sample sizes and small number of variables in the data), nonparametric tests were used to contrast the percentage of correct responses (Wilcoxon signedrank tests or Mann-Whitney $U$-tests). All $p$ values reported were two-tailed.

\section{Results}

All the children met the criteria for the test trials based on the training trials in both intramodal and cross-modal matching. Figure 1 shows the mean percentages of correct responses in intramodal and cross-modal matching for each participant.

\section{Group Differences for Two Matching Tasks}

Intramodal matching. In intramodal matching, children with ASD achieved a mean of $90.0 \%$ correct responses $(S D=8.4)$, and the children with WS achieved a mean of $85.0 \%(S D=27.2)$. There was no significant difference between the two groups (Mann-Whitney test: $Z=-0.58, p=0.589, r^{2}=0.03$ ).

Cross-modal matching. In cross-modal matching, the ASD group's mean percentage of correct responses was $44.2 \%(S D=21.8)$, and the WS group's mean was $79.2 \%(S D=19.6)$. There was a significant difference between the ASD and WS groups (Mann-Whitney test: $Z=-2.19, p=0.026, r^{2}=0.40$ ).

\section{Differences between Matching Tasks in Each Group}

For the children with ASD, percentage of correct responses in intramodal matching was higher than cross-modal matching (Wilcoxon signed-rank test: $\left.Z=-2.20, p=0.028, r^{2}=0.40\right)$. On the other hand, for the children with WS, there was no significant difference between matching tasks (Wilcoxon signed-rank test: $\left.Z=-0.73, p=0.465, r^{2}=0.04\right)$. 

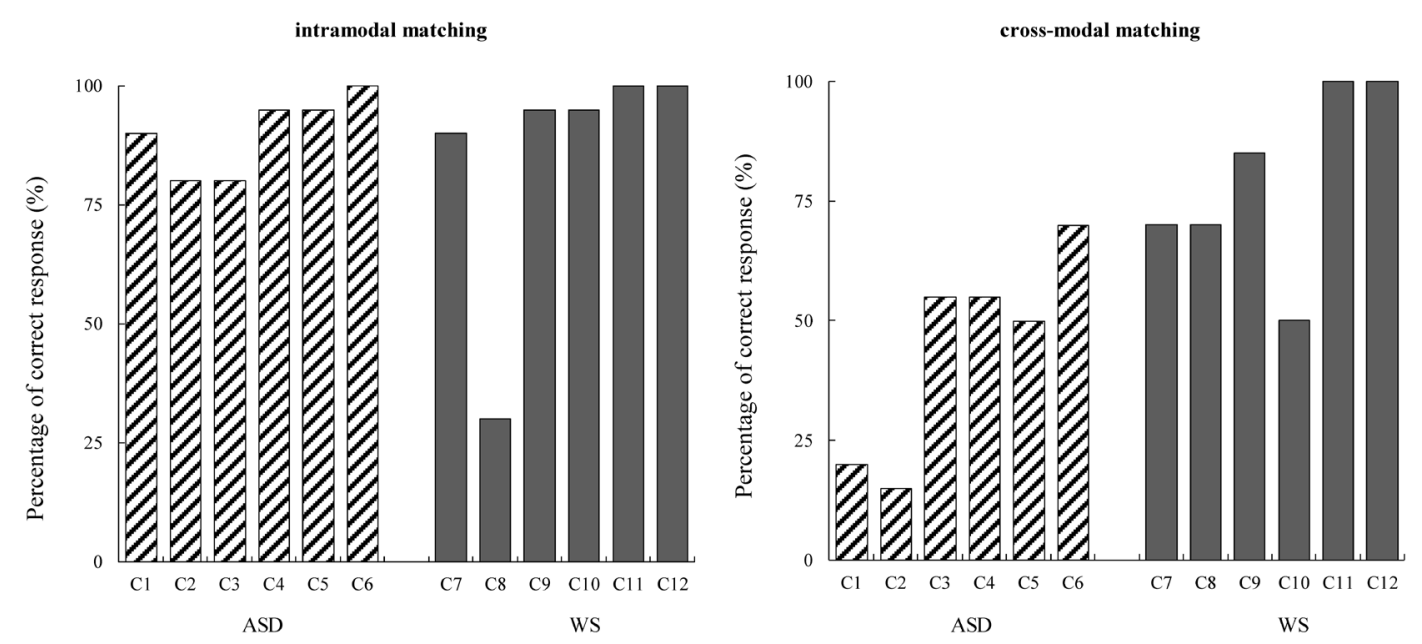

Fig. 1 Percentage of Correct Responses in the Intramodal and Cross-modal Matching Tasks for Six Children with ASD (Hatched Bars) and Six Children with WS (Gray Bars)

Note. ASD: Autism spectrum disorders; WS: Williams syndrome.

Table 2 Mean Response Rate in Cross-modal Matching for Each Sample Stimulus and Response Choice in Each Group

\begin{tabular}{|c|c|c|c|c|}
\hline \multirow{2}{*}{ Response } & \multicolumn{4}{|c|}{ Sample stimulus } \\
\hline & Happy & Surprised & Angry & Sad \\
\hline \multicolumn{5}{|c|}{ Autism spectrum disorder } \\
\hline Happy & $\underline{26.7}$ & 33.3 & 16.7 & 3.3 \\
\hline Surprised & 40.0 & $\underline{20.0}$ & 6.7 & 16.7 \\
\hline Angry & 16.7 & 30.0 & $\underline{63.3}$ & 13.3 \\
\hline Sad & 13.3 & 13.3 & 13.3 & $\underline{66.7}$ \\
\hline \multicolumn{5}{|l|}{ Williams syndrome } \\
\hline Happy & $\underline{73.3}$ & 23.3 & 6.7 & 0.0 \\
\hline Surprised & 16.7 & $\underline{66.7}$ & 6.7 & 0.0 \\
\hline Angry & 6.7 & 0.0 & $\underline{76.7}$ & 0.0 \\
\hline Sad & 3.3 & 10.0 & 10.0 & $\underline{100.0}$ \\
\hline
\end{tabular}

Note. Percentages of correct responses are underlined.

\section{Group Differences among the Four Emotions in Cross-modal Matching}

Table 2 shows the mean response rate in crossmodal matching for each sample stimulus and response choice in each group. The mean percentages of correct responses for children with ASD were $26.7 \%$ for "happy," $20.0 \%$ for "surprised," $63.3 \%$ for "angry," and $66.7 \%$ for "sad." Children with autism responded with correct answers to "happy" and "surprised" with a frequency comparable to what one would achieve by random guessing. The mean percentages of correct responses for children with WS were $73.3 \%$ for "happy," $66.7 \%$ for "surprised," $76.7 \%$ for "angry," and $100.0 \%$ for "sad." Children with WS thus performed well above the chance level on all emotions. There was no significant difference between the two groups (happy: $Z=-1.90, p=0.065$, $r^{2}=0.30$, surprised: $Z=-2.00, p=0.065, r^{2}=0.32$, angry: $Z=-1.31, p=0.240, r^{2}=0.14$, sad: $Z=-2.29, p=0.065$, $\left.r^{2}=0.44\right)$.

In Table 2, error patterns for each of the emotions in both groups are shown. We conducted 12 KruskalWallis tests with Diagnosis (ASD, WS) as an independent variable, and the percentage of errors per combination of sample stimulus and response choice as a dependent variable. None of the pairs differed significantly across diagnosis with a Bonferroni correction. There were no differences in the error patterns between the children with ASD and those with WS. 


\section{Discussion}

This study investigated the development of emotion comprehension in children with ASD and those with WS through using intramodal (visual stimulusvisual stimulus) and cross-modal (auditory stimulusvisual stimulus) matching. We used pictures of facial expressions as visual stimuli and spoken affective prosodies as auditory stimuli. The results of this study revealed that both groups showed a high percentage of correct responses in intramodal matching. On the other hand, in cross-modal matching, children with ASD showed a lower percentage of correct responses than those with WS.

This is the first study to compare children with ASD and those with WS using cross-modal matching of emotion comprehension. The results of the crossmodal matching revealed a difference in performance between the two groups. Meanwhile, the results of intramodal matching showed no difference between the groups in accordance with the results of one previous study (Lacroix et al., 2009). These findings suggest that a difference between intramodal and cross-modal matching underlies the development of emotion comprehension.

For children with ASD, cross-modal matching was more difficult than intramodal matching. This finding is consistent with other research using intramodal matching (Castelli, 2005) and cross-modal matching (Peppé et al., 2007). Deficits in emotion comprehension among children with ASD may be related to difficulties in understanding the relationship between auditory and visual stimuli.

Moreover, it is possible that children with ASD have greater difficulties in the cross-modal matching of emotion comprehension when they are younger. The participants in this study were younger (mean 5 years 9 months) and had lower developmental ages (mean 3 years 10 months) than those in previous studies. The lower percentage of correct responses in cross-modal matching may reflect the children's age. Previous research studies that used cross-modal matching are consistent with this account (Baker et al., 2010; Grossman et al., 2010; Jones et al., 2011; Linder \& Rosén, 2006; Matsuda \& Yamamoto, 2015; Paul et al., 2005; Peppé et al., 2007).

In this study, children with WS showed a high percentage of correct responses in both intramodal and cross-modal matching. This finding is consistent with previous research using intramodal matching (TagerFlusberg \& Sullivan, 2000), and it is the first example of cross-modal matching in children with WS. The results suggest that children with WS have no difficulty in understanding the relationship between auditory and visual stimuli.

There were several limitations to the study. The small sample size is the most important limitation of this study. This factor limits the statistical power of the analysis, and accordingly the findings must be considered preliminary. Thus, the replication of this study with a larger sample size will be needed. Second, this study did not investigate if participants can discriminate between affective prosodies. In future, research should include a pre assessment to confirm that participants can discriminate auditory stimuli. Third, there is no typical development group. Future studies should include a typical development group.

Despite limitations, this study demonstrates that differences between ASD and WS may result from the use of different stimuli and responses. No group difference was found in the intramodal matching, although there was a significant difference between ASD and WS in cross-modal matching. These findings contribute to our understanding of the social phenotypes of ASD and WS.

In conclusion, our results indicate a difference in the development of emotion comprehension between children with ASD and those with WS using intramodal and cross-modal matching. Children with ASD have difficulty in understanding the relationship between auditory and visual stimuli, and this difficulty may be greatest at younger ages. On the other hand, children with WS have no difficulty in understanding the relationship between auditory and visual stimuli. Based on these findings, the modality of the stimuli and responses and the child's age may have large effects on emotion comprehension in early developmental stages.

\section{Acknowledgment}

This research was supported by CREST research project on Social Imaging. Soichiro Matsuda was supported by a fellowship from the Japan Society for the Promotion of Science. 


\section{References}

American Psychiatric Association (2000) Diagnostic and statistical manual of mental disorders (4th ed. text revision). American Psychiatric Assoication, Washington, DC.

Baker, K. F., Montgomery, A. A., \& Abramson, R. (2010) Brief report: Perception and lateralization of spoken emotion by youths with high-functioning forms of autism. Journal of Autism and Developmental Disorders, 40, 123-129.

Bormann-Kischkel, C., Vilsmeier, M., \& Baude, B. (1995) The development of emotional concepts in autism. Journal of Child Psychology and Psychiatry, 36, 1243-1259.

Capps, L., Yirmiya, N., \& Sigman, M. (1992) Understanding of the emotional meaning of facial expressions in people with autism. Journal of Child Psychology and Psychiatry, 33, 11691182.

Castelli, F. (2005) Understanding emotions from standardized facial expressions in autism and normal development. Autism, 9, 428-449.

Gagliardi, C., Frigerio, E., Burt, D. M., Cazzaniga, I., Perrett, D. I., \& Borgatti, R. (2003) Facial expression recognition in Williams syndrome. Neuropsychologia, 41, 733-738.

Gross, T. F. (2008) Recognition of immaturity and emotional expressions in blended faces by children with autism and other developmental disabilities. Journal of Autism and Developmental Disorders, 38, 297-311.

Grossman, R. B., Bemis, R. H., Plesa-Skwerer, D., \& Tager-Flusberg, H. (2010) Lexical and affective prosody in children with high-functioning autism. Journal of Speech, Language, and Hearing Research, 53, 778-793.

Harms, M. B., Martin, A., \& Wallace, G. L. (2010) Facial emotion recognition in autism spectrum disorders: A review of behavioral and neuroimaging studies. Neuropsychology Review, 20, 290-322.

Ikuzawa, M., Matsushita, Y., \& Nakase, A. (2002) Kyoto scale of psychological development 2001. Kyoto International Social Welfare Exchange Centre, Kyoto. (in Japanese)

Jones, C. R. G., Pickles, A., Falcaro, M., Marsden, A. J. S., Happé, F., Scott, S. K., Sauter, D., Tregay, J., Phillips, R. J., Baird, G., Simonoff, E., \& Charman, T. (2011) A multimodal approach to emotion recognition ability in autism spectrum disorders. Journal of Child Psychology and Psychiatry, 52, 275-285.

Klein-Tasman, B. P. \& Mervis, C. B. (2003) Distinctive personality characteristics of 8-, 9-, and 10-year-olds with Williams syndrome. Developmental Neuropsychology, 23, 269-290.

Kuusikko, S., Haapsamo, H., Jansson-Verkasao, E., Hurtig, T., Mattila, M., Ebeling, H., Jussila, K., Bölte, S., \& Moilanen, I. (2009) Emotion recognition in children and adolescents with autism spectrum disorders. Journal of Autism and Develop- mental Disorders, 39, 938-945.

Lacroix, A., Guidetti, M., Rogé, B., \& Reilly, J. (2009) Recognition of emotional and nonemotional facial expressions: A comparison between Williams syndrome and autism. Research in Developmental Disabilities, 30, 976-985.

Le Couteur, A., Rutter, M., Lord, C., Rios, P., Robertson, S., Holdgrafer, M., \& McLennan, J. (1989) Autism diagnostic interview: A standardized investigator-based instrument. Journal of Autism and Developmental Disorders, 19, 363-387.

Lindner, J. L. \& Rosén, L. A. (2006) Decoding of emotion through facial expression, prosody and verbal content in children and adolescents with Asperger's syndrome. Journal of Autism and Developmental Disorders, 36, 769-777.

Lord, C., Rutter, M., DiLavore, P. S., \& Risi, S. (2002) Autism diagnostic observation schedule (ADOS). Western Psychological Services, Los Angeles.

Lord, C., Rutter, M., \& Le Couterur, A. (1994) Autism diagnostic interview-revised: A revised version of a diagnostic interview for caregivers of individuals with possible pervasive developmental disorders. Journal of Autism and Developmental Disorders, 24, 659-685.

Matsuda, S. \& Yamamoto, J. (2013) Intervention for increasing the comprehension of affective prosody in children with autism spectrum disorders. Research in Autism Spectrum Disorders, 7, 938-946.

Matsuda, S. \& Yamamoto, J. (2014) Computer-based intervention for inferring facial expressions from the socio-emotional context in two children with autism spectrum disorders. Research in Autism Spectrum Disorders, 8, 944-950.

Matsuda, S. \& Yamamoto, J. (2015) Intramodal and cross-modal matching of emotional expression in young children with autism spectrum disorders. Research in Autism Spectrum Disorders, 10, 109-115.

Paul, R., Augustyn, A., Klin, A., \& Volkmar, F. R. (2005) Perception and production of prosody by speakers with autism spectrum disorders. Journal of Autism and Developmental Disorders, 35, 205-220.

Peppé, S., McCann, J., Gibbon, F., O’Hare, A., \& Rutherford, M. (2007) Receptive and expressive prosodic ability in children with high-functioning autism. Journal of Speech, Language, and Hearing Research, 50, 1015-1028.

Plesa-Skwerer, D., Faja, S., Schofield, C., Verbalis, A., \& TagerFlusberg, H. (2006) Perceiving facial and vocal expressions of emotion in individuals with Williams syndrome. American Journal of Mental Retardation, 111, 15-26.

Plesa-Skwerer, D., Verbalis, A., Schofield, C., Faja, S., \& TagerFlusberg, H. (2006) Social-perceptual abilities in adolescents and adults with Williams syndrome. Cognitive Neuropsychology, 23, 338-349. 
Schopler, E., Reichler, R. J., DeVellis, R. F., \& Daly, K. (1980) Toward objective classification of childhood autism: Childhood autism rating scale (CARS). Journal of Autism and Developmental Disorders, 10, 91-103.

Setter, J., Stojanovik, V., van Ewijk, L., \& Moreland, M. (2007) Affective prosody in children with Williams syndrome. Clinical Linguistics \& Phonetics, 21, 659-672.

Tager-Flusberg, H., Plesa-Skwerer, D., \& Joseph, R. M. (2006) Model syndrome for investigating social cognitive and affec- tive neuroscience: A comparison of autism and Williams syndrome. Social Cognitive and Affective Neuroscience, 1, 175-182. Tager-Flusberg, H. \& Sullivan, K. (2000) A componential view of theory of mind: Evidence from Williams syndrome. Cognition, 76, 59-90.

Wallace, S., Coleman, M., \& Bailey, A. (2008) An investigation of basic facial expression recognition in autism spectrum disorders. Cognition and Emotion, 22, 1353-1380. 